\title{
Q La historia de la infancia Y EL Niño ANTE EL AÑO 2000
}

\section{Perspectiva histórica de la Educación Infantil}

Aurora Gutiérrez Gutiérez

Profesora Enterita de Historia de la Edacación

\section{Análisis de algunas propuestas de planes de estudio para la Diplomatura de Educación}

$M^{\text {"Paz Lebrero Baena }}$

Divectora del Carso de Especializacion

en Educacion Infantil

La Infancia es una calegoria histórica y social forjada conl ambivalencias.

T. Richardson

\section{PERSPECTIVA HISTÓRICA DE LA EDUCACIÓN INFANTIL.}

En el ocaso del siglo XX, cuyo comienzo parecía innovador, vamos a ver en qué medida el paidocentrismo ha sido un término que ha dado nombre a una realidad conseguida, o bien, sólo nos evidencia el flatus vocis de un siglo que ha llegado tan lejos en avances tecnológicos, y no ha conseguido realizar a ese niño que debería ser autor y señor del año 2000.

Este punto de partida nos lleva, siquiera sea someramente, a hacer un punto de inflexión en las coordenadas históricas en que nos encontramos, ya que la historia de la inlancia está, por su materia y por la dificultad práctica del registro de fuentes lidedignas, implicada en las obscuras fronteras entre las actitudes y percepciones modernas y postmodernas del futuro.

Quienes se plantean la educación de la infancia descle perspectivas de potencial para un futuro se ven abocados a una reforma estructural, porque 
como dice Gerstner ${ }^{\circ}$, uno de los más famosos ejecutivos estadounidenses, los maestros de las Escuelas del Próximo Siglo están redefiniendo sus organizaciones y reescribiendo las reglas de la profesión. Manifiesta la clara necesidad de nuevos planteamientos ya que, según el mismo autor, las Escuelas de Estados Unidos enfrentan una crisis de productividad; son costosas, ineficientes y anticuadas. Sabemos lo que cuestan, pero no conocemos el valor de lo que producen. Las escuelas públicas se miden con el patrón de lo que la sociedad gasta en ellas y no por lo que ellas dan a cambio ${ }^{7}$.

El gran fracaso de la educación sería no haber alcanzado los grandes retos, que, según Gerstner, no serían otros que: la preparación del individuo para ser ciudadano de una democracia, trabajador en una economía compleja y participante en una sociedad.

Sin embargo, creemos que poco podrá avanzar la educación si sólo nos planteamos la modificación de las estructuras desde aquello que puede afectar al niño desde fuera, es decir, en su mundo externo.

Los propios especialistas en pedagogía se han dedicado por lo común, a estudiar la organización y los planes de estudios de las escuelas y las teorias de la educación, haciendo referencia sólo ocasionalmente a lo que les sucedia a los alumnos en el hogar y en el mundo en general ${ }^{*}$.

Nos parece imprescindible, desde nuestra experiencia de logros y fracasos en la educación de la infancia, desde el análisis historiográfico sobre el niño, plantear una seria reflexión, apoyándonos en criterios autorizados que marcan nuevos caminos.

Una de las vías actuales para el estudio de la infancia, y posibles acciones educativas, es la que nos abre el mundo de la psicohistoria, con representantes en los distintos campos y aportaciones multidisciplinares.

En esta línea queremos destacar las grandes aportaciones de la medicina con Rof Carballo, psicohistoriadores de la infancia como Lloyd de Mause ${ }^{9} \mathrm{o}$ psicólogos como Pinillos.

Sintetizando los grandes rasgos de este enfoque psicohistórico, uno de los aspectos más importantes a tener en cuenta, y que debe ser considerado

\footnotetext{
"Louis V. Gerstner es el creador del Programa de las Escuelas del Próximo Siglo (Next Century Schools, patrocinado por RJR Nabisco y dedicado a la reforma educativa de la educación pública. ${ }^{7}$ Louis V. Gerstner, Jr. y otros, Reinventando la educación, Barcelona, Paidós, 1996.

${ }^{8}$ Ibid.

${ }^{4}$ Lloyd de Mause, Historias de la Infancia, Madrid, Alianza Editorial, 1992.
} 
como factor decisivo en la práctica educativa, será la resonancia afectiva en el niño. Es decir, no basta una influencia externa sobre él, sino que es necesario establecer una estrecha comunicación con la sensibilidad del niño. En la manera de tratarlo y de entenderlo, y en la reacción que despierte en el niño está la clave de la formación de la sensibilidad infantil. Y esto se define y establece en los primeros años de la vida.

Podemos repetir con Pinillos que con toda seguridad la clave del futuro, la llave que abre la esperanza en un cambio hacia el porvenir de la humanidad y que se aparte de los perennes conflictos, guerras y violencias, actividades suicidas con la capacidad que tiene el hombre de destrucción, está en la infancia, en la formación de la sensibilidad de la infancia, que es con la que ella después va a hacer frente a los problemas del mundo, y de decidir su destino y el de los demás ${ }^{10}$.

Sin embargo, desde nuestra perspectiva histórica, en la lectura que hacemos del desarrollo del niño, vemos que, con frecuencia, se minimiza el valor del niño, no considerándole como un ser sujeto y autor de significado, constructor de formas culturales ni tampoco como un actor en nuestro propio paisaje.

Pero, si bien hemos de dar un valor al niño por sí mismo, no menos interesante es conocer cómo el camino de realización el niño no lo recorre solo. Otro magnífico autor, padre de la medicina psicosómatica, el profesor Rof Carballo, ha estudiado en profundidad la realidad del niño en una relación de díada con el mundo que le circunda. A esta relación la denomina "relación transaccional" refiriéndose primordialmente al mundo de afecto que encuentra en los seres que le rodean. Esta será la trama sobre la que se tejerá la vida del niño, en cuyas pautas está ya programada su propia dimensión de adulto".

El mismo autor nos dice cómo la grandeza del ser humano radica en la profunda menesterosidad con que nace y cómo, gracias a su interacción con el adulto, según se realice -que puede ser grandiosa o miserable-, va a ser capaz de configurar su personalidad y asimilar el medio que le transmite el adulto. De cómo haya recibido en los primeros años este bagaje sociogenético va a depender su cristalización como adulto y la forma en que él lo va a transmitir y a relacionarse con los demás.

De ahí la gran importancia de cómo haya sido, a su vez, constituido el adulto para ver qué tipo de mundo puede transferir al niño, que le converti-

\footnotetext{
"JOSÉ Luis PINILlos, Introducción a la psicohistoria, "Psicohistoria de la niñez y la familia", noviembre, 1992.

${ }^{\prime}$ Rof Carballo, J.: Violencia y Ternura, Madrid, Editorial Prensa Española, 1974.
} 
rá, ya en el receptor de una riqueza para su propia configuración feliz o, de lo contrario, el niño sólo será el soporte de la gran carga frustrante del medio que recibe que no le realiza, porque en él confluyen todas las tensiones y frustraciones de la sociedad en que vive. De ahí que no quepa plantearse cómo será el niño del año 2000 sino cómo es la sociedad y el adulto que va a decidir cómo sea el niño del año 2000.

Nos encontramos, por otra parte, con la terrible paradoja de que, mientras los medios aumentan sin cesar, y la sociedad de bienestar ocupa un mayor espacio, el mundo de la infancia cada vez está más disminuido. Constantemente vemos cómo en los foros y debates en busca de soluciones a los problemas de la sociedad, con gran asombro, contemplamos la falta de espacio geográfico, psicológico y social que se concede al niño en el mundo de hoy. Los grandes debates en torno al problema del desarrollo no buscan una expansión de la sociedad, desde la consideración de la infancia. El niño es el gran excluido de un medio que sólo busca reducir la infancia para permitir vivir mejor a los que mejor viven. El niño se convierte en un ser incómodo porque exige un espacio en el bienestar de la familia, del estado y del mundo.

El análisis de una infancia cuyo desarrollo nos plantea retos no cumplidos nos lleva a nuevos replanteamientos. Es muy importante no sólo el estudio puntual de los medios, sino también recorrer el camino para llegar al conocimiento de lo que se refiere a este proceso histórico.

La preocupación más reciente por la infancia partiendo del propio niño, sobre todo de su afectividad, de su intimidad y de su realidad individual, ha convertido al niño en sujeto de la psicohistoria ${ }^{12}$.

Y sin embargo, como ha dicho un notable autor especialista de de esta etapa, Lloyd DeMause, "la historia de la infancia es una pesadilla de la que hemos empezado a despertarnos hace muy poco"; es la opinión del psicohistoriador que ha aportado un giro no sólo importante para un mejor conocimiento de la infancia sino para la búsqueda de nuevos enfoques educativos. La preocupación por la infancia, está definido en lema del Instituto de la Psicohistoria que dice: nadie entre aquí que no se interese por la infancia.

Las actitudes de suspicacia o de desconfianza, de amor o de odio, de violencia frente a la vida, concibiéndola como lugar de lucha por la existencia, en lugar de solidaridad, son valores que se desarrollan en el niño desde la proyección de los del adulto en la infancia, y desde este prisma radical es desde donde el niño va a ver la vida, teniendo las reacciones consiguientes frente al mundo.

${ }^{12}$ Pinillos, op. cit. 
Lejos de su aparente sentido de tópico, los niños son el futuro de la sociedad, pero también una realidad presente. Sin embargo, la sociedad se ha resistido a enfrentarse, a admitir una crítica que permita ver con claridad la manera de cómo se les ha preparado para hacer frente al futuro.

Y mientras hacemos grandes avances en introducir los más avanzados medios y planteamientos de un gran nivel técnico, los estudios de la historia se replantean, últimamente, lejos de hacer sólo investigación de los acontecimientos, de las monarquías, de los enlaces de dinastías, de las grandes batallas, abrir, por el contrario, el foco de la historia a los protagonistas anónimos, a la vida cotidiana, a la gente que no figura en la genealogía importante de la historia. En nuestro caso a la infancia.

Si analizamos nuestro quehacer educativo desde una perspectiva histórica, desde el punto de vista que estamos planteando, la acción educativa debe tener un enfoque de valores, de metas, más allá de sí misma.

La aportación que la psicohistoria nos da es el único sentido progresista de la vida, entendiendo como tal el desarrollo de la humanidad desde la realización de la infancia, ya que sólo desde aquí puede entenderse un progreso material y económico que parta de la realización del hombre como impulsor del progreso y no víctima del mismo.

Pero ello nos lleva a recuparar el concepto de la infancia con una visión nueva. La infancia no puede ser el contenido de aspectos carenciales y de marginación, cuya consecuencia sería la de un hombre frustrado que busque su equilibrio en la opresión y en la violencia.

Y es que no se instala uno en el campo de lo humano, en la ciencia, en los conocimientos humanos si no es desde una óptica que tenga referencia moral, desde un marco de referencia no empírico. Es decir, la acción del hombre sobre el niño se debe realizar de acuerdo con unas ciertas normas morales en las que las preferencias sean opciones libres desde una exigencia moral. Es el ejercicio de la libertad para optar por lo mejor. En educación lo óptimo debe convertirse en meta, porque en ello radica la felicidad y el verdadero nivel de vida que es el nivel del hombre.

Este sentido de una realidad matizada bajo un punto de vista moral es el que debe ser comunicado al niño en la relación con nosotros y en el proceso de interacción con los medios. Este mismo proceso de maduración y enriquecimiento, de apertura e integración del mundo en el niño va produciendo un proceso de psicologización de tipo maduro en la manera de afrontar los problemas ${ }^{13}$.

\footnotetext{
"PINIllos, op. cit.
} 
Toda referencia a lo grande, a lo supraempírico tiene que estar presente en la demarcación del objeto de la educación y del uso de los medios en la medida en que no se quiera deshumanizar la historia. Igualmente en la evolución de la niñez hay un avance lento, inexorable hacia una mayor concienciación de lo que es el niño y a un creciente avance de llegar y de acercarse a lo que es el niño, para lograr una mayor comunicación con la infancia, una mayor sensibilización desde la primera infancia. Ese camino de sensibilidad de regresión de los padres para ponerse en lugar del niño, tratar de ponerse a su altura, es una de las tesis de Lloyd de Mause.

El modelo de hombre del que partimos aqui, no es el político, ni el económico, sino el homo relatens, el hombre en busca de relación humana, ese es el modelo en el que se están buscando las claves de la regeneración de la relación humana. Las claves, por las que la relación humana puede establecerse de una forma u otra, destructiva o solidaria, los modos de subjetivación del prójimo, de la realidad, cómo ve la realidad, cómo la hace propia, cómo se sensibiliza a ello. Esos modos son el lugar de la decisión, es donde se dirime el destino solidario, o destructivo, la orientación destructiva o solidaria de ese homo relatens, de la relación humana ${ }^{\prime 4}$.

Esta visión del sentido de progreso desde valores perennes, pero no estudiados, es una alternativa al estudio de las innovaciones en los medios desde una perspectiva de la psicohistoria. Es complementaria de la historia, las fronteras de las disciplinas han caído y no se puede estudiar al niño sólo desde un aspecto educativo externo y desde fuera, sino desde un enfoque interdisciplinar.

La psicohistoria hoy ha marcado otros caminos basados en la infancia para el estudio del adulto. No podemos saber quién es un hombre sin conocer su infancia. La investigación de la infancia explica en gran parte el sentido del adulto. De aquí la proliferación del estudio de los grandes personajes a través de su biografía. Por otra parte, al adulto, principalmente a educadores y padres, incumbe la gran responsabilidad de crear ese mundo cuya trama es posible sólo en esta etapa y ofrecer aquellos medios con los que el niño pueda poner la urdimbre tan rica que constituya el adulto que quisiera haber sido.

En esta búsqueda de cómo plantearnos la educación del año 2000, nos sentimos en la obligación de recordar aquí a uno de los maestros del siglo XX. Nos referimos a Victor Frankl, judío converso de Auschwitz, nombrado doctor honoris causa por 28 Universidades y fundador de la Tercera Escuela Vienesa de Psicología. Moría el día 26 de agosto del año 1997, esta egregia figura que pasará a la historia como el profeta del sentido de la vida. Tras

${ }^{14}$ Pinillos, op. cit. 
un brillante historial,exponente de una obra imposible de mencionar en la brevedad de un artículo, queremos partir de los principios de esta gran personalidad que, desde el sufrimiento, supo encontrar los valores que dan sentido a la vida.

Para este autor, el sentido del hombre radicaría en el análisis y en la búsqueda de las verdaderas motivaciones existenciales, pero esto sólo podría realizarse desde la educación para la responsabilidad.

Sin embargo, la responsabilidad del individuo se desarrolla desde la infancia en interacción con el adulto responsable, ya que el proceso de esta realización del niño, desde los primeros años de la vida, nunca podrá darse desde una profesionalización mercantilizada donde el niño es un producto que se manufactura.

Queremos terminar esta exposición apoyándonos en el pensamiento de otro gran historiador y sociólogo que ha contribuido a enriquecer la interpretación de la psicohistoria, desde la relación dinámica entre individuo y sociedad, y que tal vez pueda ayudar a desvelar las funciones de la universidad y de la educación como función propia:

$Y$ precisamente porque el niño indefenso necesita ser modelado por la sociedad para convertirse en un ser fuertemente individualizado y diferenciado, la individualidad del adulto sólo puede ser comprendida a partir de su sentido relacional ${ }^{15}$.

\section{ANÁlisis de ALGUNAS PROPUESTAS DE PLANES DE ESTUDIO PARA LA DIPLOMATURA DE EDUCACIÓN INFANTIL}

En la actualidad, la formación inicial del profesorado de Educación Infantil está en función de los planes de estudio correspondientes a la Diplomatura en esa etapa, derivados del desarrollo de la Ley Orgánica de Reforma Universitaria de 1983, así como de los estatutos de las diferentes Universidades.

Entre los aspectos/Artículos a considerar en dicha Ley hemos de señalar: "Los estudios universitarias se estructurarán, como máximo, en tres ciclos. La superación del primero de ellos dará derecho, en su caso, a la obtención del título de Diplomado..." (Art. 30).

\footnotetext{
${ }^{15}$ Norbert Elías, La sociedad de los individuos. Barcelona, Península/ideas, 1990.
} 
“Las Universidades, en uso de su autonomía, podrán impartir enseñanzas conducentes a la obtención de otros diplomas o títulos..." (Art. 28.3).

Hemos creído interesante profundizar en el proceso seguido desde la propuesta marco del Consejo de Universidades hasta las diversas propuestas alternativas que entidades, Universidades y otros interesados en la temática han ido realizando como alternativa a aquellas propuestas. Veamos su desarrollo en los hitos más importantes del tema que nos ocupa.

\section{DOCUMENTOS LEGISLATIVOS}

Diferentes textos legislativos han ido haciendo referencia a la nueva formación y titulación de los diplomados en Educación Infantil, tratando de flexibilizar las enseñanzas universitarias y, al mismo tiempo, actualizar su curriculum a las necesidades sociales y su inserción en el mundo laboral de la Comunidad Económica Europea.

Mencionamos, a continuación, los documentos legislativos más representativos:

- El Pleno del Consejo de Universidades aprobó la Ponencia de Reforma el 28-2-87 y remitió el informe técnico elaborado por el Grupo de trabajo-Comisión 15- responsable del mismo.

Entre las directrices que figuran en el mismo caben destacarse las siguientes: denominación del título, contenidos básicos, perfil profesional, carácter de las enseñanzas, requisitos de entrada, etc. Asimismo se establecen los siguientes porcentajes de carga de las materias/asignaturas -en el sistema de créditos-.

- Debe existir entre el 30\% y 50\% de carga en materias troncales que deberán incluir todas las Universidades, a fin de dar alguna homogeneidad a todos los planes de estudio.

- En un máximo del 60\%, cada Universidad puede incorporar materias obligatorias en cada título así como otras optativas para elección del alumnado.

- Un mínimo del 10\% será de libre elección del alumnado entre las materias que se imparten.

Con el fin de promover y facilitar la participación en el debate público al personal y organismos interesados en ello, el Consejo de Universidades envió los siguientes documentos:

a) Informe técnico del Grupo de trabajo. 
b) Informe del Grupo de Trabajo en el que se podía realizar una propuesta íntegra a las presentadas en el propio título.

c) Documento en el que se podía realizar observaciones y propuestas parciales al Informe del Grupo del trabajo.

Con los documentos anteriormente mencionados, iniciamos nuestro estudio.

\section{METODOLOGÍA DEL TRABAJO}

En la realización del presente estudio hemos procedido por la lectura exhaustiva de los tomos editados por el Consejo de Universidades sobre la Reforma de las Enseñanzas Universitarias, concretamente los referidos al título de Diplomado en Educación Infantil y Primaria. De él nos hemos fijado y recogido únicamente los referentes a la Educación Infantil, objeto de este trabajo.

Una vez realizado el vaciado de los documentos, se ha elaborado una tabla por cada una de las entidades que envían las propuestas, recogiéndose en 16 tablas. Con éstas se ha elaborado una, reflejando en ella las diferencias en el número de créditos otorgados por cada entidad a las diferentes materias troncales que se proponen, como figura en nuestro Documento 1.

Por último, con todos los datos recogidos se ha realizado otra tabla comparativa (Documento 2) con las principales entidades estudiadas, Universidades y sus Departamentos, quedando fuera de la comparación algunas unidades departamentales que se consideran ya reflejadas en su correspondiente Universidad.

En la tabla aparecen materias troncales, numeradas del 1 al 35 , que reflejan los documentos estudiados. El orden en el que se presentan se deriva de la metodología empleada para realizar esta comparación, y es el siguiente:

1. Dado que se ha tomado como documento base el remitido por el Consejo de Universidades, las materias troncales que aparecen en primer lugar son las del citado documento, numeradas del 1 al 14 .

2. Se presentan las materias que proponían las diferentes entidades y que no estaban reflejadas en el documento del Consejo de Universidades. Así, desde el $n^{\prime \prime} 15$-primera materia nueva que aparece- hasta el $n^{\circ} 35$, se ha ido anotando todas las materias troncales aparecidas en los 10 documentos objeto de esta comparación.

Debido a la falta de sistematización observada en la presentación de las propuestas remitidas, se ha optado por reflejar exactamente las que 
aparecen en cada documento, aun a riesgo de repetir, como se puede observar, materias que se podrían considerar iguales (por ejemplo: Didáctica de las Matemáticas y Matemáticas, aparecidas en un mismo documento).

3. Una vez enumeradas todas las materias troncales que aparecen, y teniendo siempre como referencia el documento del Consejo de Universidades, se ha ido comparando para cada remitente estudiado el número de créditos teóricos y prácticos que se otorgan a dichas materias.

Como ya se ha apuntado, la falta de sistematización en la presentación de las alternativas hace que sólo se pueda comparar lo que estrictamente aparece en las mismas, es decir, hay remitentes que especifican que están de acuerdo con todo lo presentado en el documento aludido y añaden alguna materia nueva, pero también nos encontramos con propuestas que no especifican su acuerdo con el citado documento y sólo se limitan a reflejar, sin demasiado orden, una serie de materias con el número de créditos asignados sin desglosar en teóricos y prácticos. De este modo las materias que aparecen en la tabla son fiel reflejo de lo encontrado en los documentos estudiados y en ella se expresa con los signos $=,+,-$, según sean el mismo, mayor o menor el número de créditos asignados a las materias.

Esta comparación también se ha realizado respecto al área o áreas a las que se les asigna las materias propuestas. Las áreas que aparecen reflejadas se desglosaban hasta un número de 15 , que se han refundido en nueve, ya que se ha considerado las grandes áreas departamentales existentes; así, por ejemplo, todas las didácticas aparecidas se han agrupado en la de Didáctica y Organización Escolar.

Se acompaña al presente trabajo la especificación de las áreas que aparecen en todos los documentos estudiados, así como un listado de las materias troncales que se reflejan.

Observemos, seguidamente, los Documentos 1 y 2 a los que hemos venido haciendo alusión. 


\begin{tabular}{|c|c|c|c|c|c|c|c|c|c|c|c|c|c|c|c|c|}
\hline \multirow{3}{*}{ 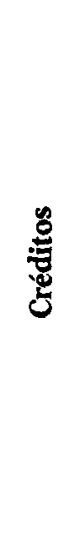 } & $\stackrel{\beth}{\beth}$ & $\widetilde{\Omega}$ & 尔 & $a$ & $a$ & さ & $\stackrel{\infty}{-}$ & & & $\underset{\stackrel{m}{\stackrel{m}{n}}}{\stackrel{m}{n}}$ & Iิ & $\begin{array}{l}2 \\
I \\
\beth\end{array}$ & $\stackrel{\infty}{=}$ & $\Xi$ & 8 & \\
\hline & $\ddot{m}$ & 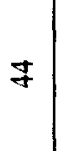 & $\subseteq$ & 0 & $a$ & $\tilde{m}$ & $\stackrel{\infty}{=}$ & & & & & & $a$ & a & 9 & \\
\hline & 8 & $\stackrel{\infty}{\infty}$ & $\stackrel{12}{\simeq}$ & $m$ & & 8 & & & & & & & $\beth$ & $\bar{\nabla}$ & $\bar{\nabla}$ & \\
\hline 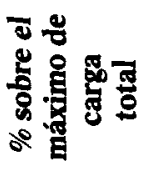 & 芷 & in & & & & 感 & & & & 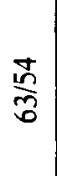 & & & & & & \\
\hline 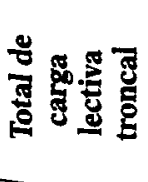 & 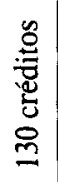 & & & & & 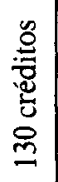 & & & & 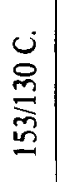 & & & & & & \\
\hline 몰 & 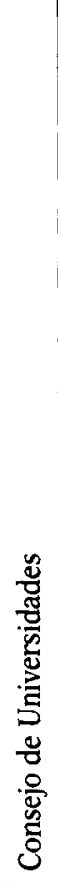 & 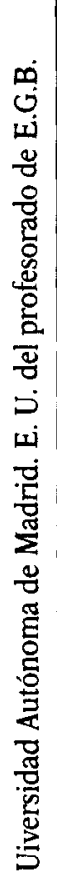 & 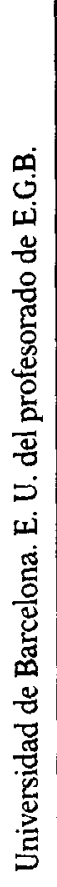 & 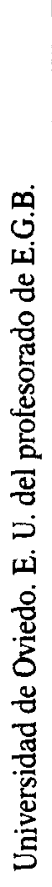 & 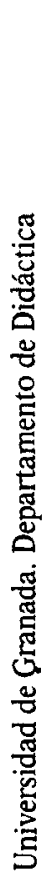 & 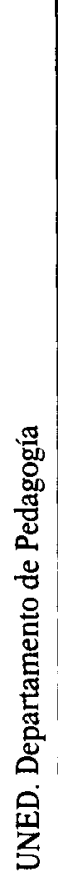 & 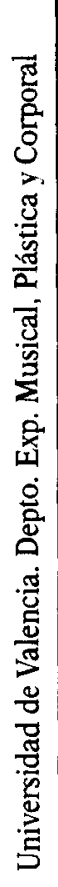 & 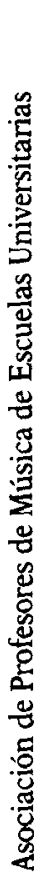 & 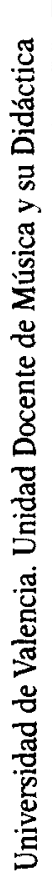 & 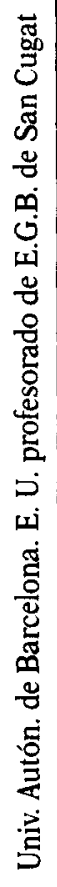 & 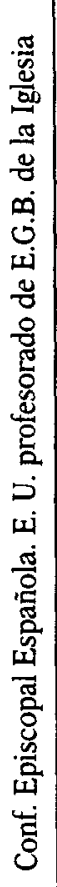 & 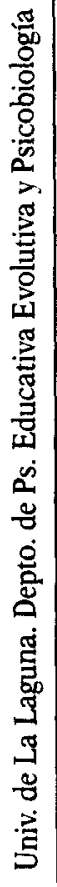 & 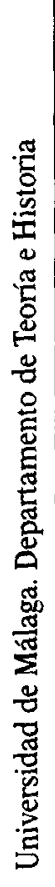 & $\begin{array}{l}\dot{\Sigma} \\
\dot{0} \\
\dot{0} \\
\dot{0}\end{array}$ & 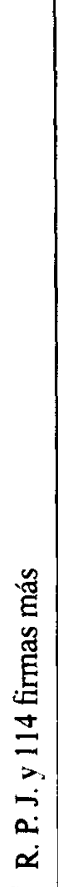 & 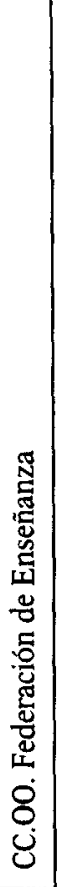 \\
\hline
\end{tabular}




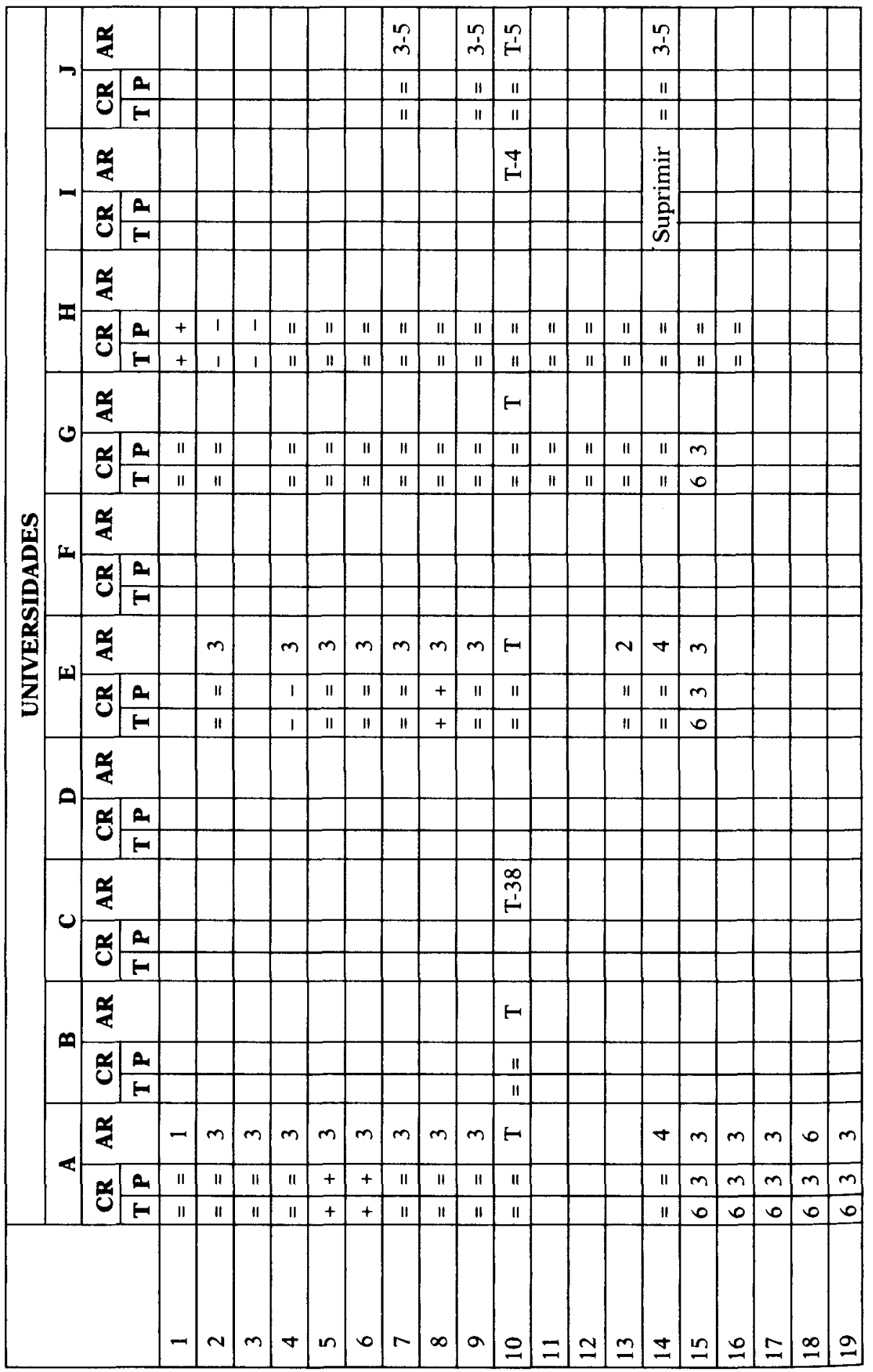




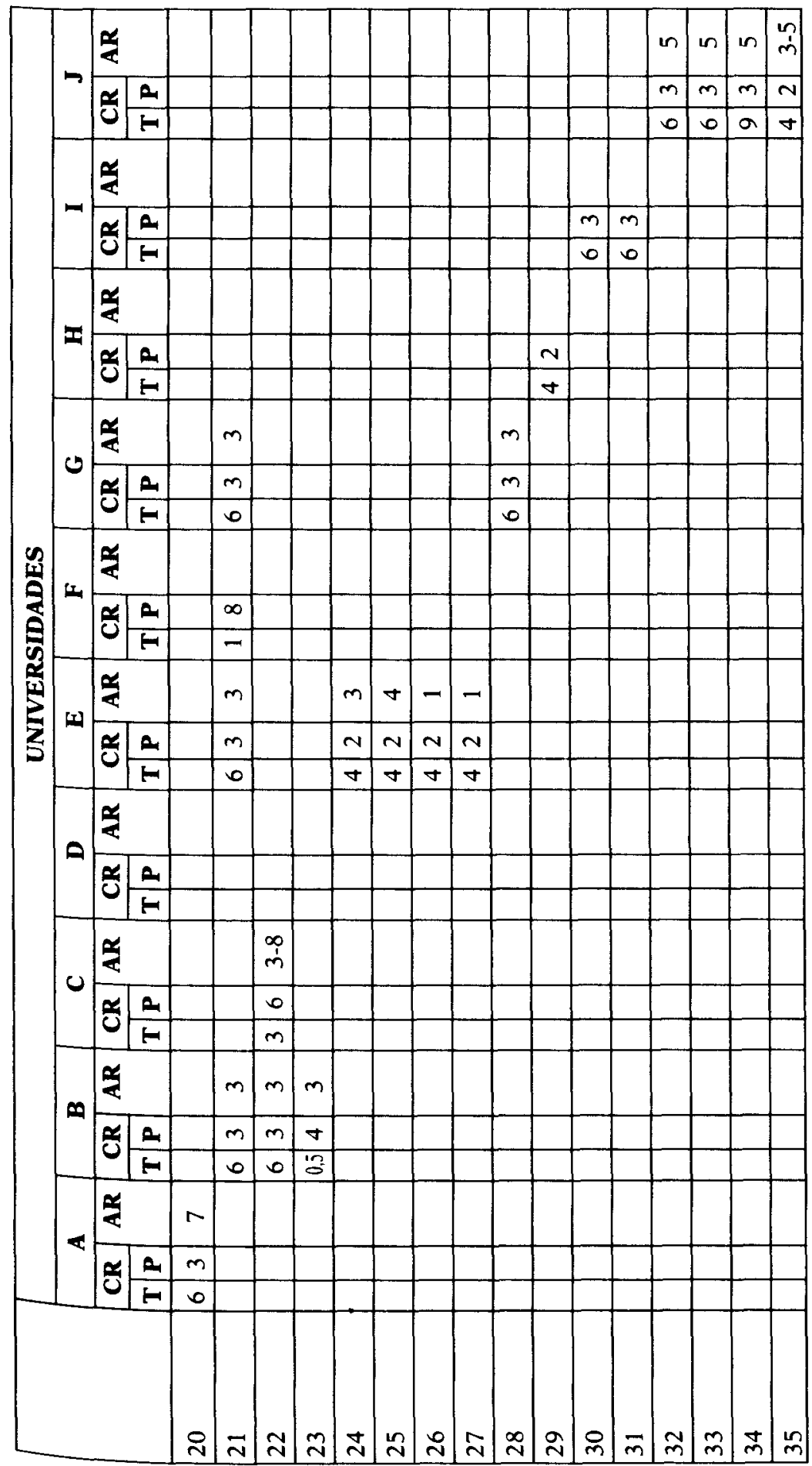




\section{LEYENDAS}

$$
\begin{aligned}
& \mathrm{CR}=\text { CRÉDITOS } \\
& \mathrm{T}=\text { TEÓRICOS } \\
& \mathbf{P}=\text { PRÁCTICOS } \\
& \mathrm{AR}=\text { ÁREAS }
\end{aligned}
$$

1. Psicología

2. Sociología

3. Didáctica y Organización Escolar

4. Teoría e Historia de la Educación

5. Métodos de Investigación y Diagnóstico en Educación (MIDE)

6. Filología Española

7. Matemáticas

8. Educación Física

9. Música

T. Todas las áreas reflejadas en el Documento presentado por el Consejo de Universidades

MATERIAS TRONCALES (ver relación)

$A=$ UNIVERSIDAD AUTÓNOMA DE MADRID

$B$ = UNIVERSIDAD DE BARCELONA

$\mathrm{C}=$ UNIVERSIDAD DE OVIEDO

D = UNIVERSIDAD DE GRANADA

$\mathrm{E}=\mathrm{UNED}$

$\mathrm{F}=$ UNIVERSIDAD DE VALENCIA

$\mathrm{G}$ = UNIVERSIDAD AUTÓNOMA DE BARCELONA

$\mathrm{H}=$ UNIVERSIDAD DE LA LAGUNA

I = UNIVERSIDAD DE MÁLAGA

$\mathbf{J}=$ NOMINAL

= IGUAL N" CRÉDITOS QUE DOCUMENTO Al (CONSEJO DE UNIVERSIDADES)

+ MAYOR N" CRÉDITOS QUE DOCUMENTO A1 (CONSEJO DE UNIVERSIDADES)

- MENOR N' CRÉDITOS QUE DOCUMENTO A1 (CONSEJO DE UNIVERSIDADES) 


\section{MATERIAS TRONCALES}

1. Bases Psicológicas de la Educación Especial

2. Didáctica de la Expresión Plástica

3. Didáctica de la Expresión Rítmica

4. Didáctica de la Lengua

5. Didáctica de las Ciencias Naturales

6. Didáctica de las Ciencias Sociales

7. Didáctica General de Ciclo

8. Educación Especial: Aspectos Didácticos y Organizativos

9. Organización del centro escolar

10. Prácticum

11. Psicología de la Educación

12. Psicología del Desarrollo

13. Sociología de la Educación

14. Teoría e Instituciones Contemporáneas de Educación

15. Didáctica de las Matemáticas (Conocimiento Lógico-Matemático)

16. Educación Ambiental

17. Educación para la Salud

18. Lengua Española

19. Infantil

20. Matemáticas

21. Didáctica de la Expresión Musical

22. Didáctica de la Educación Física

23. Formación Vocal y Auditiva

24. Didáctica de la Habilidades Lingüísticas e Instrumentales

25. Educación Infantil

26. Psicología Evolutiva y del Aprendizaje I

27. Psicología Evolutiva y del Aprendizaje

28. Didáctica de la Educación Corporal

29. Psicología del Desarrollo y Educación Afectiva y Social

30. Teoría de la Educación

31. Historia Contemporánea de la Educación y Educación Comparada

32. Bases diferenciales de la Educación

33. Metodología de la Investigación Educativa

34. Orientación y Tutoría

35. Educación Especial: Aspectos Curriculares de Diagnóstico y Tratamiento

\footnotetext{
* Hasta la 14 son las reflejadas en el Documento Al presentado por el Consejo de Universidades.
} 


\section{BIBLIOGRAFÍA}

CONSEJO DE UNIVERSIDADES: Reforma de las Enseñanzas Universitarias. Título: Diplomado en Educación Infantil y Primaria, Tomos I y Il. Madrid: Ministerio de Educación y Ciencia, Secretaría General, 1989.

ELÍAS, N. La sociedad de los individuos. Barcelona, Península/ideas, 1992.
DEMAUSE, LI. Historia de la infancia. Madrid, Alianza Editorial, 1992.

GERSTNER, V., y otros. Reinventando la educación. Barcelona, Paidós, 1996.

PINILLOS, J.L. «Introducción a la psicohistorian. Psicohistoria de la niñez y la familia, Conferencia, noviembre, 1992.

\section{RESUMEN}

En la primera parte se analiza la encrucijada del mundo de la infancia en el tránsito al segundo milenio. Implica una visión, no tanto del niño como ser aislado, cuanto del diálogo transaccional con el mundo adulto que, desde su propia jerarquía de valores, ha de realizar el niño desde los primeros años. Para ello consideramos las aportaciones de la psicohistoria que, partiendo de los valores del mundo interno de la persona, desarrollen un hombre responsable, constructor de un mundo participativo y solidario.

En la segunda parte del Artículo propuesto se trata de la importancia de la formación inicial del profesorado de Educación Infantil en la actualidad; nos ha llevado a hacer un análisis exhaustivo de las diferentes propuestas alternativas presentadas a esa Diplomatura por organismos y particulares. Comparamos variables tales como las áreas de conocimiento, las materias troncales y el número de créditos teóricos/prácticos.

Palabras clave: Crédito: En raíz anglosajona, unidad de diez horas de carga lectiva, teóricas o prácticas. Educación Infantil: En nuestro país, etapa educativa comprendida entre los 0 y 6 años. Plan de estudios: Ordenación de las enseñanzas de la carrera, con criterios y objetivos previamente marcados. 


\section{ABSTRACT}

The importance of initial training of child education teaching staff has brought, us to carry out an exhaustive analysis of the different alternative proposals submitted by organizations and individuals for this Diploma Course. We compare variables such as,.the areas of knowledge, core subjects and the number of theoreticalpractical credits.

Key words: Credit, Childhood Education, Curriculum. 